COMUNICACIÓN CIENTÍFICA IX CONGRESO NACIONAL DE LA SOCIEDAD CIENTÍFICA ESPAÑOLA DE ENFERMERÍA - SCELE. Mayo de 2018. UNIVERSIDAD DE ALICANTE.

\title{
DESARROLLO DE UN SISTEMA DE CALIDAD Y SEGURIDAD CLÍNICA EN LA UNIDAD DE REANIMACIÓN DELHGUE (Hospital General Universitario de Elche)
}

Vanessa Nolasco Guirao

Hospital General Universitario de Elche. 


\section{PALABRAS CLAVE:}

calidad, seguridad asistencial.

Objetivo general: desarrollar un sistema de gestión de calidad que satisfaga las necesidades y expectativas de todas las partes interesadas (pacientes, profesionales y accionistas).

\section{Objetivos específicos:}

Control y adecuación de la documentación escrita del servicio

Disminución de la variabilidad de la práctica clínica.

Aumento de la satisfacción de todas las partes interessadas.

Control y reducción de los eventos adversos.

Mejorar la eficiencia del servicio.

Metodología: la metodología a seguir se centra en la formación-acción trabajando al lado de los profesionales mediante la realización de seminarios/taller para el conocimiento cercano de los sistemas de gestión de la calidad.

Para el desarrollo del proyecto y el plan de trabajo se estructura el proyecto en los siguientes tres etapas :

1 - Definición del sistema de gestión de la calidad

- Determinación del contexto

- Determinación de los procesos y documentación de los mismos

- Gestión de riesgos

- Mejora de calidad e indicadores de calidad

2 - Implantación y desarrollo de los circuitos de trabajo y seguimiento de los procesos de mejora

3 - Auditoria interna y ajuste del sistema según los resultados.

\section{Implicación para la práctica clínica:}

Analizará el contexto: En el análisis del "Contexto de la organización" requiere que la unidad se evalúe a sí misma. Este análisis será realizado mediante la herramienta cualitativa DAFO. Permitiendo a lal unidad conocer la situación en la que se encuentra y cuál es el "camino" que quiere seguir.

Determinará los procesos: Clarificará las diferentes actividades de la unidad mediante la definición de un mapa de procesos. La gestión por procesos es una forma lógica de ordenar el trabajo. Un proceso se entiende como una secuencia de actividades orientadas a generar un valor añadido sobre una "entrada" para conseguir un resultado ("salida") que satisfaga los requerimientos del paciente.

El fin de la gestión por procesos es asegurar que todos los procesos del Servicio se desarrollen de forma coordinada, mejorando la efectividad y la satisfacción de todas las partes interesadas.

El sistema de Calidad ayudará a gestionar los riesgos: El "pensamiento basado en riesgos" es esencial para lograr un sistema de la calidad eficaz. Abordar tanto los riesgos como las oportunidades establece una base para aumentar la eficacia del sistema de gestión de la calidad, alcanzar mejores resultados y prevenir los efectos negativos. La gestión de riesgos será abordada con estrategias tanto proactivas (adelantándonos al suceso) mediante el análisis AMFE, como de forma reactiva (evaluando el evento adverso que ya ha sucedido), mediante el análisis CAUSA-RAIZ.

\section{Permitirá medir la calidad mediante indicadores:}

La monitorización es una actividad del sistema de gestión que busca el seguimiento periódico y sistemático de determinadas medidas que «indiquen» o «señalen» cómo de controlado está un determinado proceso o si aparecen elementos de alarma.

Para monitorizar todos los procesos a lo largo de la implantación e implementación del sistema de calidad, utilizaremos los indicadores, los cuales nos proporcionarán la información suficiente para evaluar la correcta evolución del sistema de calidad.

Limitaciones: La limitación de recursos tanto materiales como humanos. 